\title{
Software-defined laser ultrasonics: non-destructive testing
}

Klaus Hass, Lucas M. Riobo, Gerardo Gonzalez, Francisco E. Veiras, Fernando Perez-Quintian

Klaus Hass, Lucas M. Riobo, Gerardo Gonzalez, Francisco E. Veiras, Fernando Perez-Quintian, "Software-defined laser ultrasonics: nondestructive testing," Proc. SPIE 11105, Novel Optical Systems, Methods, and Applications XXII, 111050D (9 September 2019); doi: 10.1117/12.2538500

Event: SPIE Optical Engineering + Applications, 2019, San Diego, California, United States 


\title{
Software defined laser ultrasonics: non-destructive testing
}

\author{
Klaus Hass*a, Lucas M. Riobób, Gerardo Gonzalez ${ }^{\mathrm{c}}$, Francisco E. Veiras ${ }^{\mathrm{b}}$, Fernando Perez Quintian ${ }^{\mathrm{d}}$ \\ ${ }^{a}$ Universidad de Buenos Aires, GLOmAe depto de Física. Av. Paseo Colón 850, CABA, Argentina, \\ C1063ACV; bUniversidad de Buenos Aires, CONICET, GLOmAe depto de Física. Av. Paseo Colón \\ 850, CABA, Argentina, C1063ACV; 'Universidad Nacional del Comahue, Dpto de Mecánica, \\ Buenos Aires 1400, Neuquén, Argentina, 8300; ' Universidad Nacional del Comahue, Dpto de Física, \\ IITCI-CONICET, Buenos Aires 1400, Neuquén, Argentina, 8300
}

\begin{abstract}
In this work we propose and implement the use of software defined optical interferometry for the development of laser ultrasound non-destructive testing. The interferometer is conceived as an optoelectronic system that can be controlled by software. The system itself consists of five interconnected blocks: an optical system (a heterodyne interferometer), an Electrical-Optical block (a laser + an acousto-optic modulator), an Optical-Electrical block (a balanced photodetector), a software defined hardware (a software defined radio platform), and a programmable controller (a personal computer). In particular, the selected software defined hardware includes a field programmable radio frequency transceiver. On the one hand, it can synthesize the stimulus signals for the acousto-optic modulator which splits the reference and test beams. On the other hand, it can process the photo-detected high-frequency signals accordingly. This software defined radio platform not only simplifies the experimental scheme but also has such a high sensibility that provides a wide dynamic range. In order to show the performance of the system for non-destructive testing, we analyze the signals produced on aluminium plates to detect flaws in weld seams.
\end{abstract}

Keywords: Non destructive testing, laser ultrasonics, metrology, heterodyne interferometry, interferometry

\section{INTRODUCTION}

Laser ultrasonics is a widely spread technique for non-destructive testing (NDT) of metals, composite materials, ceramics, and liquids. It has one remarkable property: the material is the emitting transducer and therefore, transduction is made by light, thus eliminating any contact ${ }^{1}$. Moreover, it can inspect complex or moving surfaces in hostile environments at high temperatures since generation and detection of ultrasound are contactless.

Interferometric techniques are widely used for measuring ultrasonic waves in metals ${ }^{2,3}$. They are attractive because of their capabilities of measuring sub-picometer displacements of high speed phenomena ${ }^{4}$. Among the variants of design for this type of applications there are homodyne $e^{3,4,5,6}$ and heterodyne ${ }^{7,8,9}$ interferometers. In the case of homodyne interferometers, active stabilization mechanisms must be used to achieve the maximum sensitivity or a highly time-consuming post processing signal is required. Moreover, in the detection electronics, sensitivity at low

frequencies is compromised by the $1 / \mathrm{f}$ noise. These limitations are overcome by heterodyne interferometers, at the expense of greater complexity in the design of the optoelectronic subsystems involved that generally require a several $\mathrm{MHz}$ frequency detection range ${ }^{10}$. We have shown different implementations of heterodyne interferometers using software defined radio (SDR) platforms to modulate and demodulate the optical signals involved in the system ${ }^{8,9}$. In particular, these SDR platforms not only simplify the experimental scheme but also relax the design restrictions in the modulation, detection and demodulation electronics. This can be possible by harnessing the very high sensitivity and wide dynamic range of the SDR platforms, since their original purpose is for implementing telecommunication systems. However, SDR platforms platforms are already found in specific applications beyond telecommunications. This lead us to propose a general architecture for the implementation of software-defined optoelectronic systems (SDO) ${ }^{11}$. The SDO concept refers to optoelectronic systems in which most of the functionality associated with signal conditioning and processing is digitally implemented and controlled by software. This includes the optical signal modulation and demodulation, and coding and decoding, with minimum hardware modifications. We believe that laser ultrasonics could

Novel Optical Systems, Methods, and Applications XXII, edited by Cornelius F. Hahlweg, Joseph R. Mulley, Proc. of SPIE Vol. 11105. 111050D · @ 2019 SPIE 
exploit the advantages of the SDO architecture (for example in ${ }^{12,13}$ ) due to its instrumentation flexibility. In this work, we introduce the development of laser ultrasound non-destructive testing by means of software defined optical interferometry. In the following sections, we show our experimental setup for NDT. Then, we demonstrate its capabilities by analyzing the interferometric signals obtained with aluminium plates. Finally, we present the conclusions.

\section{EXPERIMENTAL SETUP}

The system itself consists of five interconnected blocks: an optical system (a heterodyne interferometer), an electrical-optical block (a laser + an acousto-optic modulator), an optical-electrical block (a balanced photodetector), a software defined hardware (a software defined radio platform), and a programmable controller (a personal computer). The complete setup is arranged in an optical breadboard that is easily transported (Figure 1).

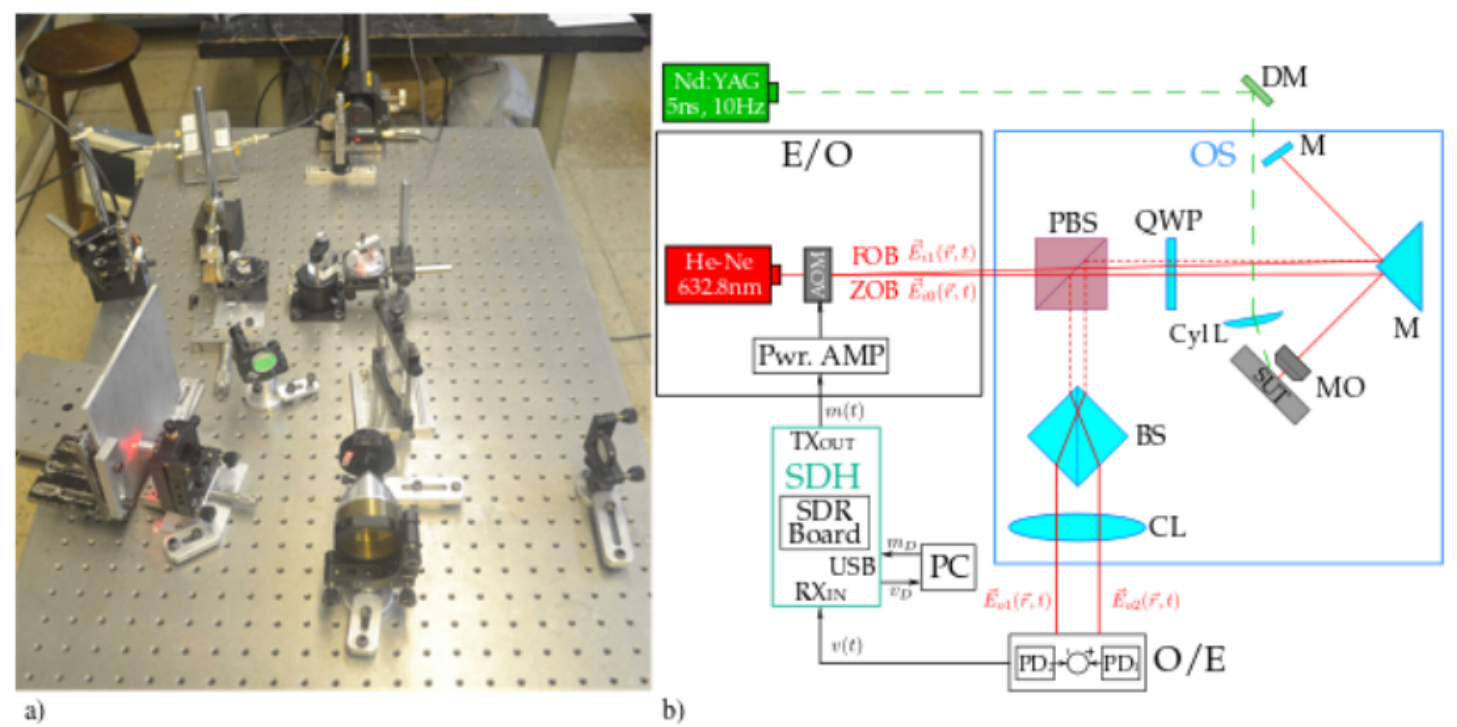

Figure 1.Figure 1. a) Picture of the experimental setup. b) Scheme of the experimental setup.

\subsection{The electrical optical block (E/O)}

This block consists of a vertically polarized continuous wave (CW) laser source, an acousto-optic modulator (AOM) and an RF amplifier. This block receives a modulating analog electrical signal $\mathrm{m}(\mathrm{t})$ that inputs the RF amplifier (IntraAction model ME-701S) which drives the AOM. Then, the AOM difracts and shifts the frequency of the impinging CW laser. Therefore, the AOM splits the incident beam into a reference beam (zero-order beam, ZOB) and a frequency shifted test beam (FOB) . We adjust the angle of the AOM in order to obtain balanced intensities between both output beams.

\subsection{Balanced path heterodyne interferometer (OS)}

The optical system (OS) receives the reference and test beams and delivers two pi-shifted interferograms to the O/E containing the information of the out of plane displacement of the sample. The input beams, ZOB and FOB, traverse almost the same path (Figure $1 \mathrm{~b}$ ). Both beams are transmitted through a polarizing beam splitter (PBS) and a quarter wave plate (QWP) oriented at 45 degrees from the vertical and then separated by a sharp-edge two mirror mount (SM). The FOB (test beam) is focused into the aluminium plate under study (SUT) by means of a microscope objective (MO) whereas the ZOB (reference beam) is reflected on a reference plane mirror (PM). After respective reflections, both beams traverses the QWP again, thus their polarization plane rotates 90 degrees and therefore they get reflected by the PBS (almost no return radiation to the laser). Then, both beams enter a non-polarized beam splitter (BS) that recombines them and produce two pi-phase-shifted interferograms ${ }^{14}$. The aluminium samples are fixed to a three-axis mount that 
permits alignment and translation. The pulsed excitation is provided by a doubled Nd-YAG laser (Continuum Minilite, $\lambda_{\text {pulsed }}=532 \mathrm{~nm}$ ) that is focused by means of a tilted mirror (DM) and a cylindrical lens (CylL) onto the samples.

\subsection{The optical-electrical (O/E) block}

This block converts the phase shifted interferograms into electrical signals and delivers an analog electrical signal v(t) to the SDH block. As a balanced photodetector, it eliminates the DC component and also common mode noise (mainly due to laser source instabilities). It consists of two reversed biased photodiodes (SFH2701) followed by a transimpedance amplifier (TIA) based on a high-speed Op-Amp (OPA847) (Figure 2). The characteristics of this block verified by the methodology exposed $\mathrm{in}^{15}$ resulting in a $2 \mathrm{kohm}$ transimpedance gain and a $200 \mathrm{MHz}$ bandwidth. The output electrical impedance of the TIA is adjusted 50 ohms in order to match the input impedance of the SDH block.
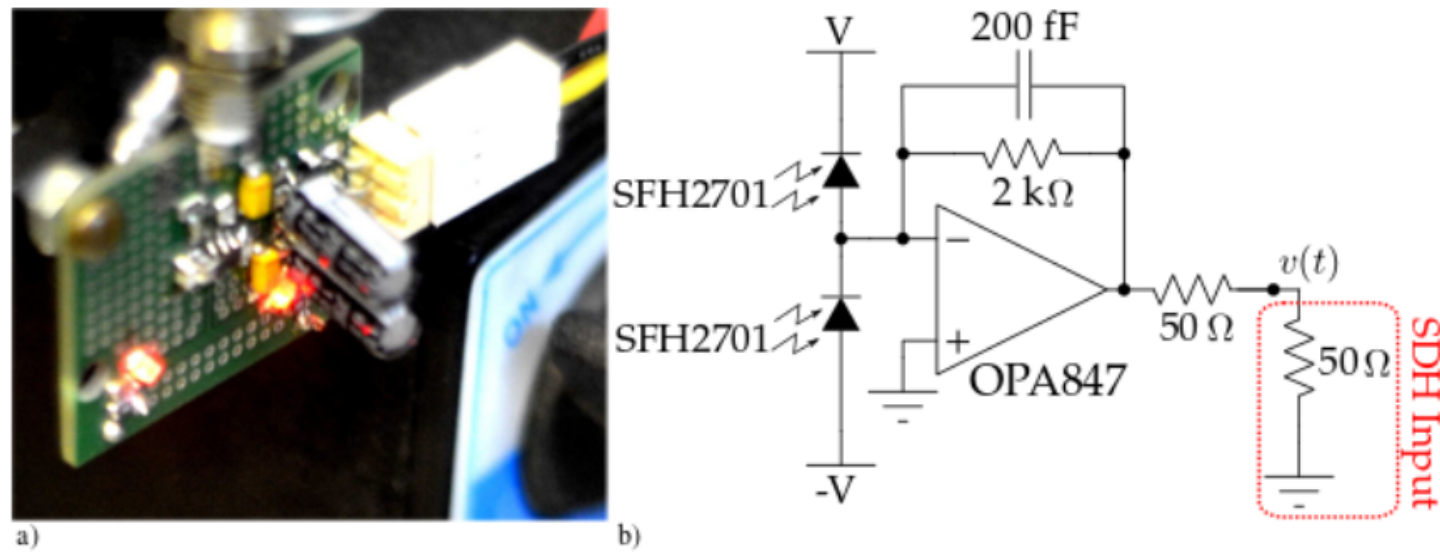

Figure 2. a) Picture of the balanced ad-hoc photodetector. b) Circuit diagram.

\subsection{The software defined hardware (SDH)}

The selected software defined hardware is based on a software defined radio (SDR) platform ${ }^{16}$ that includes a programmable radio frequency transceiver. This platform can transmit and receive signals from $100 \mathrm{kHz}$ to $3.8 \mathrm{MHz}$. It integrates 12-bit ADCs and DACs, LNAs, filters and mixers in order to provide two transmit and receive paths. Signals can be IQ modulated and demodulated to baseband ${ }^{11}$.

Here, we take advantage of the parallelism between the stimulus-response characterization method and the communications problem. In the latter, the receiver must estimate the unknown transmitted message through a noisy, dispersive and possibly time-varying channel. In the stimulus-response characterization problem, the channel characteristics must be estimated through the comparison of the (known) transmitted and received signals.

In this work, we set just a single carrier frequency to $70 \mathrm{MHz}$, as a known transmitted signal, in order to maximize the efficiency of our AOM. Then, the aluminium plate, excitated by the pulsed laser, adds a phase modulation (time varying channel) due to the induced displacement on its surface. Therefore, the received signals are phase modulated and the SDH delivers an IQ data stream that contains the displacement information.

This SDR platform clearly simplifies the experimental scheme by replacing the usually required generator and oscilloscope. Moreover, since it is capable of detecting radio communications signals, it also provides such a high sensibility and a wide dynamic range. 


\subsection{Signal processing}

We record 15 shots for each experimental condition in order to promediate the signal and reduce the uncertainty of the measurement. Here, the time stamp is provided by the pulsed laser radiation. Each signal is recovered from the interferograms difference $\mathrm{v}(\mathrm{t})$ with a spectrum around the carrier frequency. The SDH brings the signal to baseband and delivers a stream of IQ data that can be processed in order to obtain the displacement $\mathrm{d}$ by simply

$$
d=\lambda_{C W} \frac{1}{4 \pi} \arctan \left(\frac{L}{Q}\right)
$$

where $\lambda_{C W}$ is the wavelength of the CW laser $(632.8 \mathrm{~nm})$ and I,Q corresponds to the in-phase and quadrature signals respectively.

\section{EXPERIMENTAL RESULTS}

We analyze several aluminium (AA1100) welded plates that present different flaws and three plates presumably without flaws. All the plates were $5 \mathrm{~mm}$ thick, $150 \mathrm{~mm}$ wide and $150 \mathrm{~mm}$ long.

The ultrasound (US) was generated by focusing the laser beam into a line (using a cylindrical lens) at five different points (P1 to P5), the detection point (D) was located at $30 \mathrm{~mm}$ from the welding seam and points P1 to P5 were $10 \mathrm{~mm}$, $20 \mathrm{~mm}, 40 \mathrm{~mm}, 50 \mathrm{~mm}$ and $60 \mathrm{~mm}$ from D, as illustrated in Figure 3 a). Therefore, at each generation point (P1 to P5) part of the wave travels towards the detection point $\mathrm{D}$ and part of the wave travels in the opposite direction. The signals obtained at point D by the laser impinging at P2 for two representative samples are shown in Figure 4 (a). The first arrival of the ultrasonic waves is clearly noted in both signals. The waves travel at $2940 \mathrm{~m} / \mathrm{s}$ and their shapes are consistent with Rayleigh waves produced partly in the ablation regime and partly in thermoelastic regime ${ }^{2}$.

When the US is generated at points P1 or P2 the part of the wave that travels in the direction opposite to point D can be reflected at the welding seam. The amplitude of this reflected wave is dependent on the quality of the welding: as quality increases, the amplitude of the reflected wave decreases. The signal corresponding to sample 3 shows a noticeable reflected Rayleigh wave and sample 8 shows no reflected wave at all. The scheme of Fig. 3 (b) shows the two parts of the generated wave (black arrows) and the reflected wave (yellow arrow).

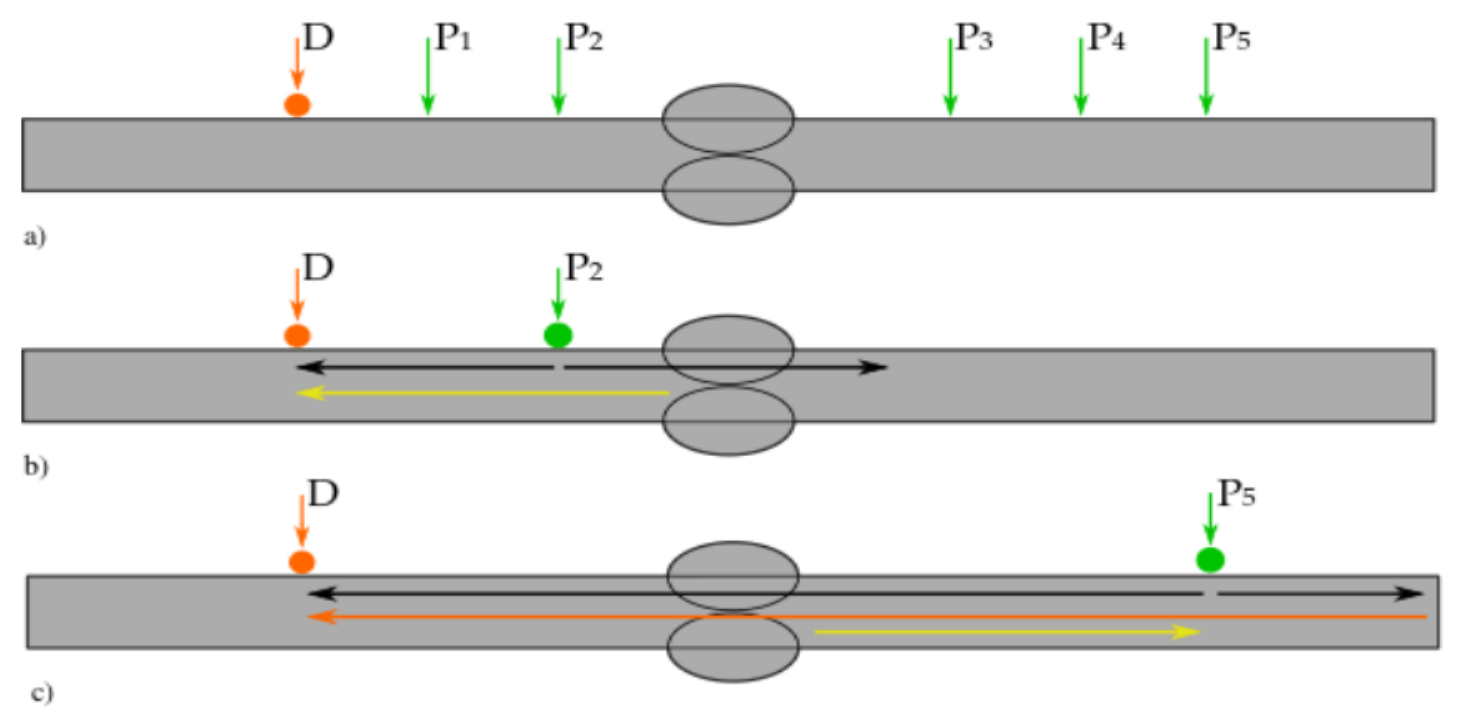

Figure 3.Scheme of the Aluminium sample under study. (a) Points of incidence of the pulsed laser (P1 to P5) and point of incidence of the CW laser (D). (b) US waves associated with pulsed laser radiation impinging on point P2. (c) US waves associated with pulsed laser radiation impinging on point P5. 
When the US comes from generation at points P3, P4 or P5, again, one part of the wave travels through the weld and is detected at point $\mathrm{D}$. The other part travels in opposite direction until it reaches the edge where it is reflected towards D through the weld. Therefore, depending on the quality of the weld, the wave reflected at the edge can also be detected at point D. Figure 3 (c) schematize the waves generated by the pulsed laser at point P5 (black arrows) and the wave reflected at the edge (orange arrow). If there are no flaws in the seam, a second wave must be detected, correspondent to this reflection. If there is some defect in the weld, the wave could be reflected again (yellow arrow) and it might decrease the amplitude of the second detected wave. Therefore, in this case, the better the quality the higher the amplitude of the wave reflected at the edge.

Figure 4 (b) shows the signals obtained at $\mathrm{D}$ by the laser impinging at $\mathrm{P} 4$ for the same two representative samples shown in Fig 4; sample 3 and sample 8. Now, the absence of the reflected wave at the edge is consistent with the presence of a defect in sample 3.

We tested several samples with and without defects that exhibit the characteristics mentioned above. In some samples this behaviour is not as notorious as in others, for them a metallographic analysis must be used to elucidate the cause of these differences.

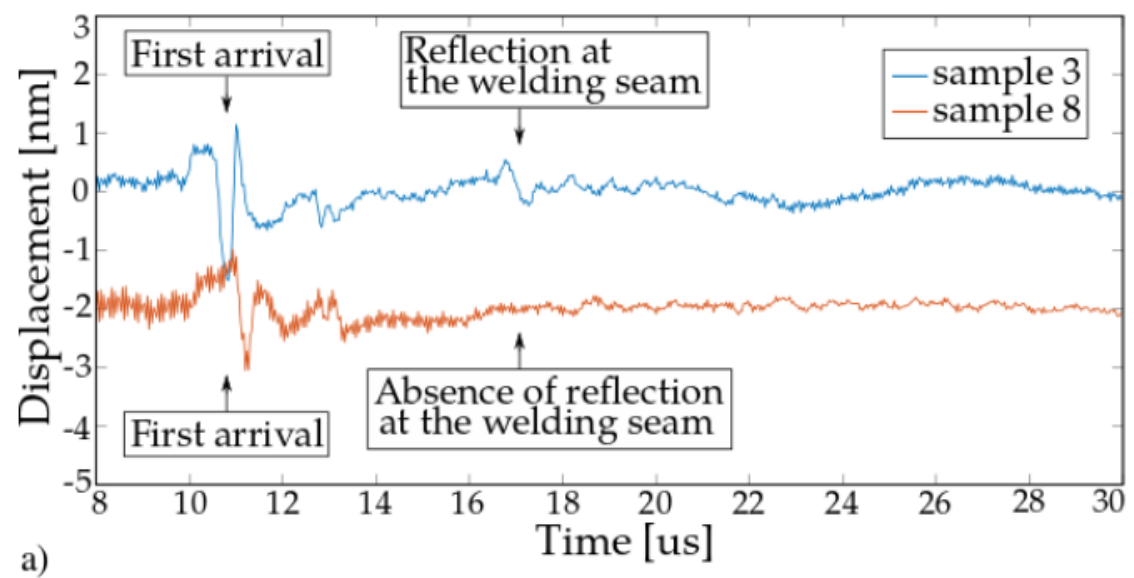

a)

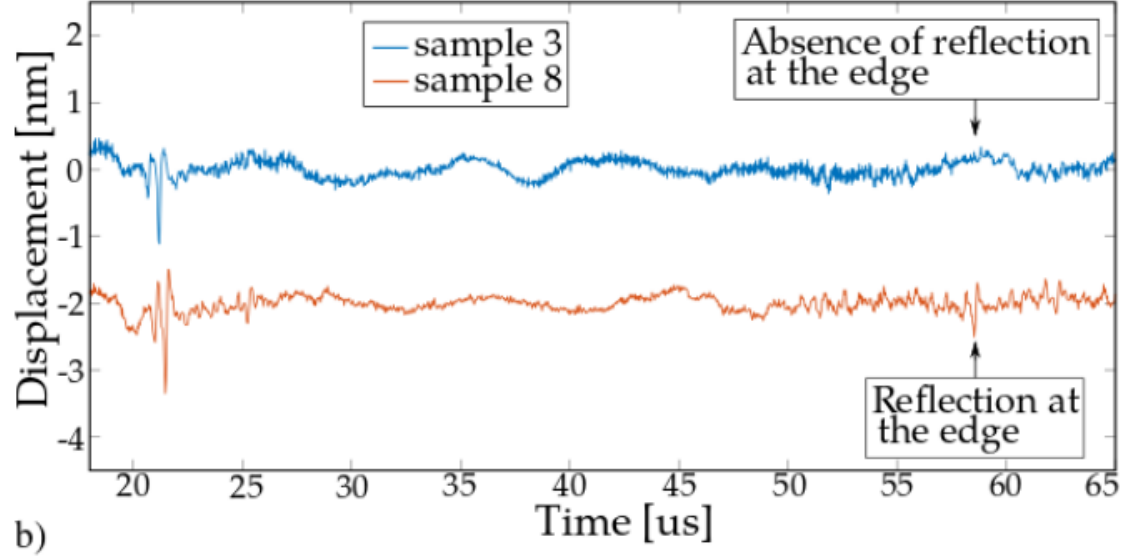

Figure 4. US (displacement in $\mathrm{nm}$ vs time in seconds) signals obtained at D for pulsed laser impinging at (a) P2 and (b) P4. 


\section{CONCLUSIONS}

Heterodyne interferometers are very sensitive systems capable of detecting high speed, laser-induced ultrasonic displacements in metals. As many instrumentation optoelectronic systems, their implementation involves strict design conditions, potentially making the system less flexible for different applications. Here, we presented a different approach on implementing heterodyne interferometric systems, namely software-defined optical interferometers. This design architecture involves the use of software-defined hardware (SDH) which can modulate and demodulate the optical signals involved, by means of conveniently designed electrical-optical (E/O) and optical-electrIcal blocks (O/E). The whole process is defined by software, so the interferometer can change its functionality by software modifications only. In particular, we used a software-defined radio platform which gives the advantage to relax the design conditions of both $\mathrm{E} / \mathrm{O}$ and $\mathrm{O} / \mathrm{E}$ blocks. This software defined hardware not only allow us to replace typical RF instrumentation equipment (for example, an RF signal generator and a wide-band oscilloscope) required for implementing heterodyne interferometers but also to obtain an elevated sensibility.

In order to show the performance of the system for non-destructive testing, we detected and analyzed laser-induced ultrasonic signals on aluminium plates to detect flaws in weld seams. Moreover, we believe that this methodology could also be applied to the determination of material properties.

\section{FUNDING INFORMATION}

This work is supported by two UBACYT grants from Universidad de Buenos Aires UBACyT 2018-2019 20020170200232BA, UBACyT 2017-2019 20020160100042BA, a grant from Universidad Nacional del Comahue (UNCo) PIN 2018 04/I241, and a grant from ANPCyT (PICT 2016-2204).

\section{REFERENCES}

[1] Monchalin, J. P. Laser-ultrasonics: principles and industrial applications. In Ultrasonic and Advanced Methods for Nondestructive Testing and Material Characterization ,pp. 79-115, (2007).

[2] C. B. Scruby and L. E. Drain. [Laser ultrasonics: techniques and applications] CRC Press, (1990).

[3] N. Alvarez, M. T. Garea \& F. Perez Quintián. "Ultrasonic Lamb waves detection using an unstabilized optical interferometer with uncalibrated phase shifts”, Journal of Modern Optics, 62:7, 556-559 (2015) .

[4] Pisani, M. "A homodyne Michelson interferometer with sub-picometer resolution". Measurement Science and Technology, 20(8), 084008 (2009).

[5] Zhu, J., Hu, P., \& Tan, J. "Homodyne laser vibrometer capable of detecting nanometer displacements accurately by using optical shutters.” Applied optics, 54(34), 10196-10199 (2015).

[6] Riobó, L. M., Veiras, F. E., Sorichetti, P. A., \& Garea, M. T. "Wideband quad optical sensor for high-speed sub-nanometer interferometry.-2 Applied optics, 56(3), 397-403 (2017)..

[7] Hitchman, S., van Wijk, K., Broderick, N., \& Adam, L. "Heterodyne interferometry for the detection of elastic waves: a tutorial and open-hardware project.” European Journal of Physics, 36(3), 035011(2015).

[8] Riobó, L. M., Veiras, F. E., Garea, M. T., \& Sorichetti, P. A. "Applications of software defined radio to heterodyne optoelectronics”. In Frontiers in Optics (pp. JTu2A-23). Optical Society of America.(2017)

[9] Riobo, L. M., Veiras, F. E., Gonzalez, M. G., Garea, M. T., \& Sorichetti, P. A. "High-speed real-time heterodyne interferometry using software-defined radio.” Applied optics, 57(2), 217-224 (2018).

[10]Ellis, J. D., \& Society of Photo-optical Instrumentation Engineers. [Field guide to displacement measuring interferometry.] Bellingham, UK: SPIE Press (2014).

[11] Riobó, L. M., Veiras, F. E., Garea, M. T., \& Sorichetti, P. A. "Software-Defined Optoelectronics: Space and Frequency Diversity in Heterodyne Interferometry.” IEEE Sensors Journal, 18(14), 5753-5760.(2018). 
[12] Ryzy, M., Grabec, T., Österreicher, J. A., Hettich, M., \& Veres, I. A. "Measurement of coherent surface acoustic wave attenuation in polycrystalline aluminum.” AIP Advances, 8(12), 125019 (2018).

[13] Kellnberger, S., et al. "Optoacoustic microscopy at multiple discrete frequencies." Light: Science \& Applications 7.1: 109 (2018).

[14] J. A. Ferrari and E. M. Frins. "Single-element interferometer, Optics Communications." vol. 279, no. 2, pp. 235-239, (2007).

[15] Riobó, L. M., Veiras, F. E., Garea, M. T., \& Sorichetti, P. A. "Wideband Transimpedance Amplifiers for Optoelectronics: Applications to Dynamic Interferometry.” Revista elektron, 1(1), 16-22 (2017).

[16] LimeMicrosystems. https://limemicro.com/products/boards/limesdr/

*khass@fi.uba.ar; phone +54 1152850429 